\title{
Chemotherapy e-prescribing: opportunities and challenges
}

This article was published in the following Dove Press journal:

Integrated Pharmacy Research and Practice

21 May 2015

Number of times this article has been viewed

\author{
Khaled A Elsaid ${ }^{1,2}$ \\ Steven Garguilo' \\ Christine M Collins ${ }^{2}$ \\ 'Department of Pharmaceutical \\ Sciences, School of Pharmacy, MCPHS \\ University, Boston, MA, ${ }^{2}$ Pharmacy \\ Services, Rhode Island Hospital, \\ Providence, RI, USA
}

\begin{abstract}
Chemotherapy drugs are characterized by low therapeutic indices and significant toxicities at clinically prescribed doses, raising serious issues of drug safety. The safety of the chemotherapy medication use process is further challenged by regimen complexity and need to tailor treatment to patient status. Errors that occur during chemotherapy prescribing are associated with serious and life-threatening outcomes. Computerized provider order entry (CPOE) systems were shown to reduce overall medication errors in ambulatory and inpatient settings. The adoption of chemotherapy CPOE is lagging due to financial cost and cultural and technological challenges. Institutions that adopted infusional or oral chemotherapy electronic prescribing modified existing CPOE systems to allow chemotherapy prescribing, implemented chemotherapy-specific CPOE systems, or developed home-grown chemotherapy electronic prescribing programs. Implementation of chemotherapy electronic prescribing was associated with a significant reduction in the risk of prescribing errors, most significantly dose calculation and adjustment errors. In certain cases, implementation of chemotherapy CPOE was shown to improve the chemotherapy use process. The implementation of chemotherapy CPOE may increase the risk of new types of errors, especially if processes are not redesigned and adapted to CPOE. Organizations aiming to implement chemotherapy CPOE should pursue a multidisciplinary approach engaging all stakeholders to guide system selection and implementation. Following implementation, organizations should develop and use a risk assessment process to identify and evaluate unanticipated consequences and CPOE-generated errors. The results of these analyses should serve to further enhance the chemotherapy electronic prescribing process and improve the quality and safety of cancer care.
\end{abstract}

Keywords: computerized provider order entry, electronic health records, chemotherapy

\section{Introduction}

Pharmacological management of cancer has undergone a significant evolution in the past decade with the introduction of targeted biologic agents and diffusion of orally available chemotherapies, along with continued use of the traditional infusional agents. As a result, contemporary treatment regimens have increased in complexity and are likely tailored to patients' characteristics and prognosis. Adverse drug events and toxicities remain significant for chemotherapy agents due to their inherent toxicities, low therapeutic indices, and vulnerability of cancer patients. Oncology drug safety is further challenged by the potential for errors during the different phases of the medication use process, ie, prescribing and transcribing, dispensing, administration, and monitoring. The chemotherapy medication error rate among hospitalized patients is not well documented. However, among ambulatory patients, estimates of chemotherapy
Correspondence: Khaled A Elsaid Department of Pharmaceutical Sciences, School of Pharmacy, MCPHS University, 179 Longwood Avenue, Boston, MA 021I5, USA

Email khaled.elsaid@mcphs.edu (c) (i) (5) 2015 Elsaid et al. This work is published by Dove Medical Press Limited, and licensed under Creative Commons Attribution - Non Commercial (unported, v3.0) License. The full terms of the License are available at http://creativecommons.org/licenses/by-nd/3.0/. Non-commercial uses of the work are permitted without any further permission fove Medical Press Limited, provided the work is properly attributed. Permissions beyond the scope of the License are administered by Dove Medical Press Limited. Information on how to request permission may be found at: http://www.dovepress.com/permissions.php 
medication errors can range between $7.1 \%$ for adult patients and $18.8 \%$ for pediatric patients, with the majority of errors occurring in the administration phase. ${ }^{1}$ Chemotherapy medication errors that occur in the prescribing phase appear to be associated with more significant adverse outcomes, with approximately one-third of all identified errors classified as serious or life-threatening. ${ }^{2}$

Health care organizations have implemented technologies, eg, computerized order entry, wireless infusion pumps, and automated dispensing systems, and embraced a culture of safety and error reporting as part of a multimodal strategy to enhance the safety of the medication use process. ${ }^{3,4}$ A national survey of pharmacy practice in hospital settings in the USA reported that automated dispensing and barcodeassisted medication administration are used in $89 \%$ and $50 \%$ of hospitals, respectively. ${ }^{5}$ Additionally, electronic health records (EHR) systems are available in 67\% of hospitals. ${ }^{5}$ Implementation of computerized provider order entry (CPOE) systems in hospital settings has dramatically increased, with $4 \%$ of hospitals reporting they used a CPOE system in 2005 compared with over 90\% in 2014. 5,6 As part of the meaningful use initiative, and in response to the US government's incentive to accelerate the rate of adoption of information technology in the health care sector, hospital acquisition of at least a basic EHR system has increased from $12 \%$ in 2009 to $59 \%$ in $2013.6,7$ A similar trend in implementing EHR systems is also observed in the ambulatory care setting. ${ }^{6}$

CPOE systems have been extensively evaluated for their effectiveness in reducing preventable adverse drug effects and medication errors in ambulatory and hospital settings. In a recent meta-analysis, hospital-based CPOE implementation was associated with a significant reduction in risk of preventable adverse drug reactions (relative risk 0.47 ; 95\% confidence interval [CI] $0.31-0.71)$, and medication errors (relative risk $0.46 ; 95 \%$ CI $0.35-0.60$ ) compared with paper-based prescribing. ${ }^{8}$ In the ambulatory care setting, CPOE implementation was associated with a significant reduction in risk of prescribing errors (odds ratio 0.30; $95 \%$ CI 0.23-0.40) compared with paper-based prescribing. ${ }^{9}$ The use of CPOE for chemotherapy prescribing appears to be lagging, with few hospitals successfully using CPOE for complex chemotherapy prescribing. ${ }^{10}$ The reluctance to adopt chemotherapy CPOE is understandable due to: increased awareness of errors and unintended consequences of CPOE; ${ }^{11-13}$ use of complex drug combinations with critical administration route use/avoidance, dosing frequency, and duration; and need for multidisciplinary review and documentation. ${ }^{10}$

\section{Methods}

We evaluated published reports describing the process of implementing infusional or oral chemotherapy electronic prescribing and summarized the reported effects of electronic prescribing on outcomes related to medication safety. The Medline (January 1, 1996 through January 22, 2015), EMBASE (January 1, 1996 through January 22, 2015), and CINAHL (January 1, 1996 through January 22, 2015) databases were searched for the terms "computerized order entry" or "computerized provider order entry" plus "chemotherapy" or "chemotherapy electronic prescribing". We included studies for further analysis if they were obtainable in full text and written in the English language.

\section{Results \\ Designing and implementing infusional chemotherapy electronic prescribing}

There are few reports in the literature describing the implementation of infusional chemotherapy electronic prescribing in an ambulatory and/or inpatient care setting and reporting on outcomes of the implementation. Table 1 summarizes the types of electronic chemotherapy prescribing and the specific safety and clinical decision support (CDS) features described in these reports. The approach to chemotherapy electronic prescribing can be broadly classified into: modifying organization-wide CPOE systems to incorporate chemotherapy electronic prescribing; acquisition of a standalone commercially available chemotherapy electronic prescribing module; or in-house development of an electronic chemotherapy prescribing module.

In a substantial number of these reports, the authors discussed implementation of an intervention in the form of a CPOE modification to allow electronic prescribing of chemotherapy regimens. ${ }^{10,14-16}$ The commercially available CPOE system was part of an organization-wide EHR system. The integration of chemotherapy prescribing into an EHR system infrastructure allowed real-time access to patient information, including laboratory values, vital signs, treatment parameters, and documentation of chemotherapy administration. ${ }^{16}$ Electronic prescribing of chemotherapy based on an EHR system infrastructure generated opportunities to evaluate the utilization of chemotherapy agents for safety and costcontainment monitoring purposes. ${ }^{16}$ Electronic prescribing of chemotherapy in this setting resulted in improving safe and 
Table I Implementation of infusional chemotherapy prescribing in ambulatory and inpatient care settings

\begin{tabular}{|c|c|c|c|}
\hline Reference & Setting & $\begin{array}{l}\text { Form of chemotherapy electronic } \\
\text { prescribing }\end{array}$ & $\begin{array}{l}\text { Safety and clinical decision support } \\
\text { features }\end{array}$ \\
\hline Meisenberg et al ${ }^{14}$ & $\begin{array}{l}\text { Mixed ambulatory patients and } \\
\text { inpatients in the USA }\end{array}$ & $\begin{array}{l}\text { Modification of an existing CPOE system } \\
\text { by programming chemotherapy regimens. }\end{array}$ & $\begin{array}{l}\text { CPOE is part of the EHR system. } \\
\text { CDS drug-drug interaction, duplicate } \\
\text { therapy, and dose limit alerts. }\end{array}$ \\
\hline Elsaid et $\mathrm{a}^{21}$ & $\begin{array}{l}\text { Mixed ambulatory patients and } \\
\text { inpatients in the USA }\end{array}$ & $\begin{array}{l}\text { Institutionally developed online, readily } \\
\text { accessible, modifiable regimen-specific } \\
\text { electronic prescribing templates. }\end{array}$ & $\begin{array}{l}\text { Automatic dose calculations based on BSA } \\
\text { and AUC. } \\
\text { Dosage adjustment for toxicities, renal and } \\
\text { hepatic functions. }\end{array}$ \\
\hline Hoffman et al ${ }^{10}$ & $\begin{array}{l}\text { Mixed pediatric ambulatory and } \\
\text { inpatient setting in the USA }\end{array}$ & $\begin{array}{l}\text { Modification of an existing CPOE system } \\
\text { by programming chemotherapy regimens. }\end{array}$ & $\begin{array}{l}\text { CPOE is part of EHR system. } \\
\text { CDS drug-drug interaction, duplicate } \\
\text { therapy, and dose limit alerts. }\end{array}$ \\
\hline $\begin{array}{l}\text { Harshberger et al }{ }^{15} \\
\text { Brockstein et al }{ }^{16}\end{array}$ & $\begin{array}{l}\text { Mixed ambulatory and inpatient } \\
\text { setting in the USA }\end{array}$ & $\begin{array}{l}\text { Modification of an existing CPOE system } \\
\text { by programming chemotherapy regimens. }\end{array}$ & CPOE is part of the EHR system. \\
\hline Small et al ${ }^{18}$ & Ambulatory setting in the UK & $\begin{array}{l}\text { Commercially available chemotherapy } \\
\text { electronic prescribing program. }\end{array}$ & Automatic dose calculation and adjustment. \\
\hline $\begin{array}{l}\text { Huertas } \\
\text { Fernández et al }{ }^{19}\end{array}$ & Inpatient setting in Spain & $\begin{array}{l}\text { Commercially available chemotherapy } \\
\text { electronic prescribing computer program. }\end{array}$ & $\begin{array}{l}\text { Automatic dose calculation and adjustment. } \\
\text { Instructions on chemotherapy preparation } \\
\text { and administration are provided. }\end{array}$ \\
\hline Kim et al ${ }^{17}$ & $\begin{array}{l}\text { Mixed pediatric ambulatory and } \\
\text { inpatient setting in the USA }\end{array}$ & $\begin{array}{l}\text { Modification of an existing CPOE system } \\
\text { by programming chemotherapy regimens. }\end{array}$ & $\begin{array}{l}\text { Preprogrammed order sets with automatic } \\
\text { dose calculations. }\end{array}$ \\
\hline Voeffray et $\mathrm{al}^{20}$ & $\begin{array}{l}\text { Mixed ambulatory and inpatient } \\
\text { setting in Switzerland }\end{array}$ & $\begin{array}{l}\text { Institutionally developed chemotherapy } \\
\text { electronic prescribing computer program. }\end{array}$ & Automatic dose calculation and adjustment. \\
\hline
\end{tabular}

Abbreviations: AUC, area under the curve; BSA, body surface area; CDS, clinical decision support; CPOE, computerized provider order entry; EHR, electronic health records.

timely drug administration through real-time treatment plan update and verification. ${ }^{16}$ Additionally, CDS was designed and implemented in these systems in the form of alerts, eg, dose limit, drug-drug interactions, and duplicate therapy alerts. Investigators developed alerts that were deemed clinically significant, and in some instances, physicians could only override the alert if a reason was entered. Kim et al described their organization's approach to modifying a CPOE system to allow prescribing of a complex chemotherapy regimen through programming of prespecified order sets. ${ }^{17}$ These regimen-specific order sets list standardized chemotherapy doses and allow the prescribing of adjunctive supportive care treatments. The CDS features included dose calculations and adjustment based on relevant patient data and clinical laboratory values. ${ }^{17}$ In this study, chemotherapy CPOE was not a component of an organization-wide EHR system, yet the safety and CDS features seemed comparable with those reported in studies where CPOE was part of an EHR system.

Another approach to implementing electronic chemotherapy prescribing was via the acquisition of commercially available chemotherapy-specific prescribing computer systems. ${ }^{18,19}$ Small et al reported on the implementation of commercially available oncology prescribing software in an ambulatory care setting. ${ }^{18}$ Prior to implementation of the prescribing software, customization of prescribing templates and menus was performed by specialist oncology pharmacists in the institution. While the oncology prescribing software was equipped with CDS features including dose calculations, adjustments, and alert generation, the software did not interface with other patient health record systems in the hospital. This lack of integration was cited as a factor contributing to suboptimal use of the prescribing software. Huertas Fernández et al discussed their institution's experience in implementing a commercially available chemotherapy prescribing software in an inpatient setting. ${ }^{19}$ As a result of the implementation, clinical workflow was reorganized to include pharmacist validation of physicians' electronic prescriptions, resulting in improvement of the safety of the chemotherapy medication use process.

The development of an in-house electronic prescribing intervention, using commercially available software programs, was adopted by two groups of investigators. Voeffray et al described the development of a prescribing module directly connected to a standardized chemotherapy regimen database. Upon entry of the patient's data (eg, height, weight, serum creatinine) by the physician, chemotherapy doses were automatically computed. The prescribing module allowed dose modification and required physicians to enter a reason for dose modification. ${ }^{20}$ The prescribing module 
was electronically linked to pharmacy and nursing systems, which allowed the electronic validation process to occur as well as documentation of preparation and administration of chemotherapy. Elsaid et al reported the development of chemotherapy regimen-specific prescribing templates. Following entry of the patient's data (eg, height, weight, serum creatinine), chemotherapy doses were automatically calculated. ${ }^{21}$ Prescribers were able to select the dosing intensity of any chemotherapy drug from a list of prespecified evidence-based dosing levels. Additionally, a dose adjustment level was preprogrammed into the prescribing module and the adjusted dose was automatically calculated. The prescribing module also contained supportive care instructions as well as administration instructions. Completed chemotherapy prescriptions were automatically sent to the pharmacy for validation and preparation of chemotherapy. In their design, the chemotherapy electronic prescribing program did not interface with other patient health records systems.

Customizing an existing CPOE system or development of an in-house prescribing module requires significant programming effort and expertise, an investment of human resources that is not required when implementing commercially available chemotherapy-specific prescribing programs, and may not be readily available in all care settings. Additionally, standardization of chemotherapy protocols, a necessary step in the development of customized chemotherapy prescribing templates, often involves multidisciplinary teams of physicians, nurses, and pharmacists with multiple rounds of critical evaluation and optimization. Use of chemotherapy CPOE integrated in an organization-wide EHR system provides the highest level of CDS and prospective safety surveillance among all the chemotherapy electronic prescribing approaches discussed in the literature. Due to significant financial costs, not all institutions can implement an organization-wide EHR system. Interfacing a chemotherapy prescribing-enabled CPOE system with other clinical information systems in the hospital can enhance the safety of chemotherapy prescribing and review by permitting realtime access to patients' relevant clinical data by physicians, pharmacists, and nurses.

\section{Outcomes of infusional chemotherapy electronic prescribing}

The outcomes of implementing infusional chemotherapy electronic prescribing are presented in Table 2. Meisenberg et al adopted a quasi-experimental sequential study design to compare handwritten orders, preprinted orders, and CPOE orders over a 5-year period for the frequency of problems or errors in chemotherapy orders. ${ }^{14}$ Examples of order problems included missing or incomplete patient identifiers and height, weight, or clinical laboratory values. Examples of chemotherapy errors included wrong dose or dose calculation, unapproved drug abbreviations, chemotherapy, and supportive care omissions. Investigators observed a significant reduction in the probability of order problems and errors when the institution switched to using preprinted order forms from handwritten orders. Additionally, a significant reduction in order problems and errors was observed in CPOE-generated orders compared with preprinted order sets. Use of CPOE completely eliminated problems and errors related to missing patient identifiers and data, and erroneous dose calculations. These error types have persisted when switching from handwritten to preprinted order sets. The authors further identified new types of errors that were unique to $\mathrm{CPOE}$ prescribing, and may occur with any CPOE system. A significant number of these errors may lead to chemotherapy overdosing through failure to reduce chemotherapy doses in all future cycles of a chemotherapy regimen in response to a significant toxicity. Some chemotherapy agents are used in multiple regimens, with a wide range of dosing intensities. Therefore, a single-dose maximum limit was not feasibly programmed for all agents. The authors identified prescribing errors due to mistyping by physicians that were classified as drug overdose errors.

Elsaid et al used an interrupted time series analysis with segmented regression analysis to compare the rate of prescription errors in the pre-implementation, implementation, and post-implementation phases of electronic prescribing. ${ }^{21}$ There was a progressive and significant reduction in risk of prescribing errors when transitioning to chemotherapy electronic prescribing. Errors of dosing calculations were most common during the pre-implementation phase. Chemotherapy electronic prescribing was associated with a significant reduction in risk of dosing calculation errors (relative risk 0.06; 95\% CI 0.03-0.1). Calculation errors that might have resulted in drug overdosage were further assessed by a trained oncologist, and errors occurring in the post-implementation phase were judged to be of lower severity compared with errors in the pre-implementation or implementation phases.

Harshberger et al evaluated the impact of chemotherapy electronic prescribing using EHR/CPOE on completeness of regimen documentation for six chemotherapy regimens and clinical staff satisfaction. ${ }^{15}$ Documentation of patient data, treatment plan, number of cycles, and administration 
instructions were used to calculate an overall regimen completeness score. EHR/CPOE was associated with an average completeness score of $92 \%$ compared with $67 \%$ for handwritten paper orders. Associated with the improvement in completeness of regimen documentation was an improvement in satisfaction among nurses, pharmacists, and physicians, with the biggest improvement in satisfaction seen among physicians. Small et al compared prescription error rates using either a spreadsheet-prescribing program or CPOE.$^{18}$ While spreadsheets could be computer-generated, they required manual input of key data and dosage adjustments. These functions were performed automatically in the chemotherapy CPOE system. Implementation of the CPOE system was associated with a $42 \%$ reduction in overall error rate. Dose calculation errors were specifically reduced, with a risk of errors using spreadsheets of $6.8 \%$ compared with $1.9 \%$ using CPOE. Interestingly, while the overall error rate was reduced with $\mathrm{CPOE}$, there was a greater number of errors of high severity associated with CPOE compared with spreadsheets. The increase in errors of high severity with CPOE was regimen-specific, and might have been partly due to the process by which the CPOE system was implemented.

Huertas Fernández compared prescription error rates in handwritten versus computerized chemotherapy prescriptions using a concurrent observational study design. ${ }^{19}$ Use of chemotherapy $\mathrm{CPOE}$ was associated with a significant reduction in overall prescription errors. CPOE use resulted in enhanced documentation, with elimination of errors of omission compared with handwritten prescriptions. Kim et al investigated the impact of CPOE implementation on the chemotherapy process in the form of completeness and accuracy of data that had the potential for high morbidity or mortality potential if left uncorrected in a pediatric setting. ${ }^{17}$ The critical process steps that were assessed included treatment plan correctness, order correctness, dose calculations accuracy, treatment plan and patient match and nurse review. CPOE implementation was associated with a significant reduction in risk of errors in the form of improper dosing, order, treatment plan, or nurse review. Unanticipated by the investigators, implementation of CPOE was associated with a significant increase in the likelihood of mismatching specific chemotherapy drug doses to specific protocols. The increase in this type of prescription error was attributed to a user interface problem. Voeffray et al studied the impact of CPOE implementation on prescription error rates. ${ }^{20} \mathrm{CPOE}$ implementation was associated with a significant reduction in overall error rate. The biggest impact of CPOE implementation was in reduction of errors linked to chemotherapy preparation and dose calculation. CPOE implementation resulted in the emergence of a new type of drug administration error that was not present with handwritten prescription. This was attributed to a lack of documentation of the presence of a central venous device in the patient's profile in CPOE. This lack of documentation resulted in altering the duration of infusion of certain chemotherapy agents, eg, vincristine or doxorubicin.

\section{Oral chemotherapy: current practices and risk of harm}

The number of orally available chemotherapy agents has significantly increased in the past decade, with more than 60 agents currently approved in the USA. ${ }^{22}$ Additionally, $20 \%-30 \%$ of chemotherapy agents in the development pipeline will be administered orally. ${ }^{23}$ Recent studies of the oral chemotherapy medication use process identified key vulnerabilities, including patient education about drug handling and adverse effects, prescription writing, monitoring, and toxicity management, and highlighted the need to adopt rigorous safety standards. ${ }^{24-26}$ However, adoption of these practice standards has been lagging for the oral chemotherapy agents. In a survey of major comprehensive cancer centers in the USA, Weingart et al identified variable practices in prescribing, monitoring, and education of patients receiving oral chemotherapy, and recommended development of oral chemotherapy safety standards. ${ }^{27}$ Variations in oral chemotherapy practices and lack of formal policies have also been recently identified across cancer centers in Canada. ${ }^{28}$

Variability in adoption of strict safety standards may be linked to the significant risk of medication errors associated with prescribing, dispensing, administration, and monitoring of oral chemotherapy drugs. A recent study analyzed reports of oral chemotherapy medication errors compiled from multiple sources, including published reports, web searches, data from comprehensive US cancer centers, and clinician reports, among other sources. ${ }^{29}$ While most of the reported oral chemotherapy errors were categorized as near misses, $20 \%$ of errors were associated with an adverse drug event. An analysis of 508 oral chemotherapy error reports revealed that approximately $50 \%$ of errors were at the prescribing stage of the medication use process. ${ }^{29}$ Additionally, the most frequently encountered type of medication error was "wrong dose" followed by "wrong drug". ${ }^{29}$ One particular oral chemotherapy agent that is associated with a high risk of medication error is oral methotrexate, given its routine use in chronic autoimmune disorders, eg, rheumatoid arthritis. In 
total, $37 \%$ of reported oral methotrexate medication errors were categorized as errors in the prescribing stage and were associated with serious adverse events. ${ }^{30}$

\section{Oral chemotherapy electronic prescribing: types and outcomes}

The implementation and outcomes of electronic prescribing for oral chemotherapy agents is described in two published reports. ${ }^{25,31}$ Table 3 compares and contrasts the nature of the oral chemotherapy electronic prescribing modules and the specific safety and CDS features in these modules. Collins and Elsaid modified an existing CPOE system to enable prescribing of oral chemotherapy agents. ${ }^{25}$ Specialized oral chemotherapy order sets, including recommended monitoring laboratory orders, were developed and programmed into the CPOE system. Access to the oral chemotherapy prescribing module was restricted to attending oncologists and physicians who prescribe oral chemotherapy agents for non-chemotherapy indications, eg, rheumatologists. CDS functionalities included programmed drug-specific maximum dose alerts and frequency options, minimum defaults for dosing and frequency, entry fields for protocol number, and treatment cycle. Concurrent with the development of oral chemotherapy CPOE, drug-specific guidelines were developed and linked to the oral chemotherapy CPOE module.

Weingart et al described safety enhancements that were introduced to an EHR system to allow electronic prescribing of oral chemotherapy. ${ }^{31}$ These enhancements included the ability to prescribe oral chemotherapy agents in either

Table 2 Outcomes of infusional chemotherapy electronic prescribing interventions in hospital and ambulatory care settings

\begin{tabular}{lll}
\hline Reference & Study design & Outcomes of interest \\
\hline Meisenberg et al ${ }^{14}$ & Quasi-experimental sequential design & Problem rate (eg, missing patient information, inappropriate \\
comparing handwritten orders, & supportive care measures) \\
& preprinted order sets, and CPOE & Error rate (eg, wrong dose calculation, frequency, drug or \\
& prescribing & supportive treatment omission)
\end{tabular}

Elsaid et $\mathrm{a}^{21} \quad \begin{aligned} & \text { Quasi-experimental design in the } \\ & \text { form of interrupted time series with } \\ & \text { segmented regression }\end{aligned}$

$\begin{array}{ll}\text { Hoffman et al } & \text { No formal study was conducted } \\ \text { Harshberger et al }{ }^{15} & \text { Retrospective chart review }\end{array}$

Brockstein et $\mathrm{al}^{16}$

Small et al ${ }^{18}$

Quasi-experimental design comparing error rates in chemotherapy prescriptions using spreadsheet templates versus using CPOE

Huertas Two-arm observational study

Fernández et al ${ }^{19} \quad$ comparing error rates in handwritten and computerized prescriptions
Monthly prevented prescribing errors per I,000 chemotherapy doses during the pre-implementation, implementation, and post-implementation phases

Reporting of patient safety events related to $\mathrm{CPOE}$ Chemotherapy documentation completeness in EHR/CPOE and handwritten orders of select chemotherapy regimens Satisfaction of clinical staff with EHR/CPOE and handwritten orders

Error rate using spreadsheet chemotherapy prescriptions versus error rate using CPOE

Error rate using handwritten prescriptions versus CPOE prescriptions

Correct completion rates of steps of high importance as determined by oncologists, eg, proper medication dose for the regimen, correct dose calculation, and nurse review documentation handwritten prescriptions versus CPOE prescriptions

Voeffray et $\mathrm{al}^{20} \quad$ Before and after comparing error rates in handwritten prescriptions versus CPOE prescriptions

Abbreviations: $\mathrm{Cl}$, confidence interval; $\mathrm{CPOE}$, computerized provider order entry; EHR, electronic health records; N/A, not available; RR, relative risk. 
fixed dose, weight-based, or body surface area-based dosing schemes. The CPOE system uses the most recent values for patient's weight and height and can be updated by prescribers. The oral prescribing module was programmed to trigger dose limit alerts if daily or weekly maximum cumulative doses are reached. Specific entry fields for intent of therapy and cancer diagnosis, cycle days, and protocol numbers were also created. Some of these entry fields were not mandatory, eg, the intent of therapy and diagnosis. The CPOE module required a complete prescription for successful transmission.

Table 4 summarizes the safety outcomes of electronic prescribing of oral chemotherapy. The implementation of oral chemotherapy electronic prescribing improved the safety of the medication use process and reduced prescribing errors. Collins and Elsaid reported a significant reduction in the odds of a prescribing error associated with oral chemotherapy CPOE, with an approximate reduction in the risk of prescribing errors of $69 \%{ }^{25}$ The authors categorized prescribing errors as any unintended significant reduction in the probability of treatment being timely and effective or an increase in the risk of harm when compared with generally accepted practices. ${ }^{32,33} \mathrm{In}$ addition to reducing the risk of prescribing errors, electronic prescribing of oral chemotherapy was associated with elimination of dosing limit errors. In the pre-implementation phase, oral methotrexate accounted for approximately $46 \%$ of errors that could lead to patient harm. Oral chemotherapy CPOE eliminated methotrexate-linked errors.

Weingart et al studied the utilization of prescription entry fields following implementation of the enhanced oral chemotherapy prescribing module. ${ }^{31}$ Physicians' utilization of entry

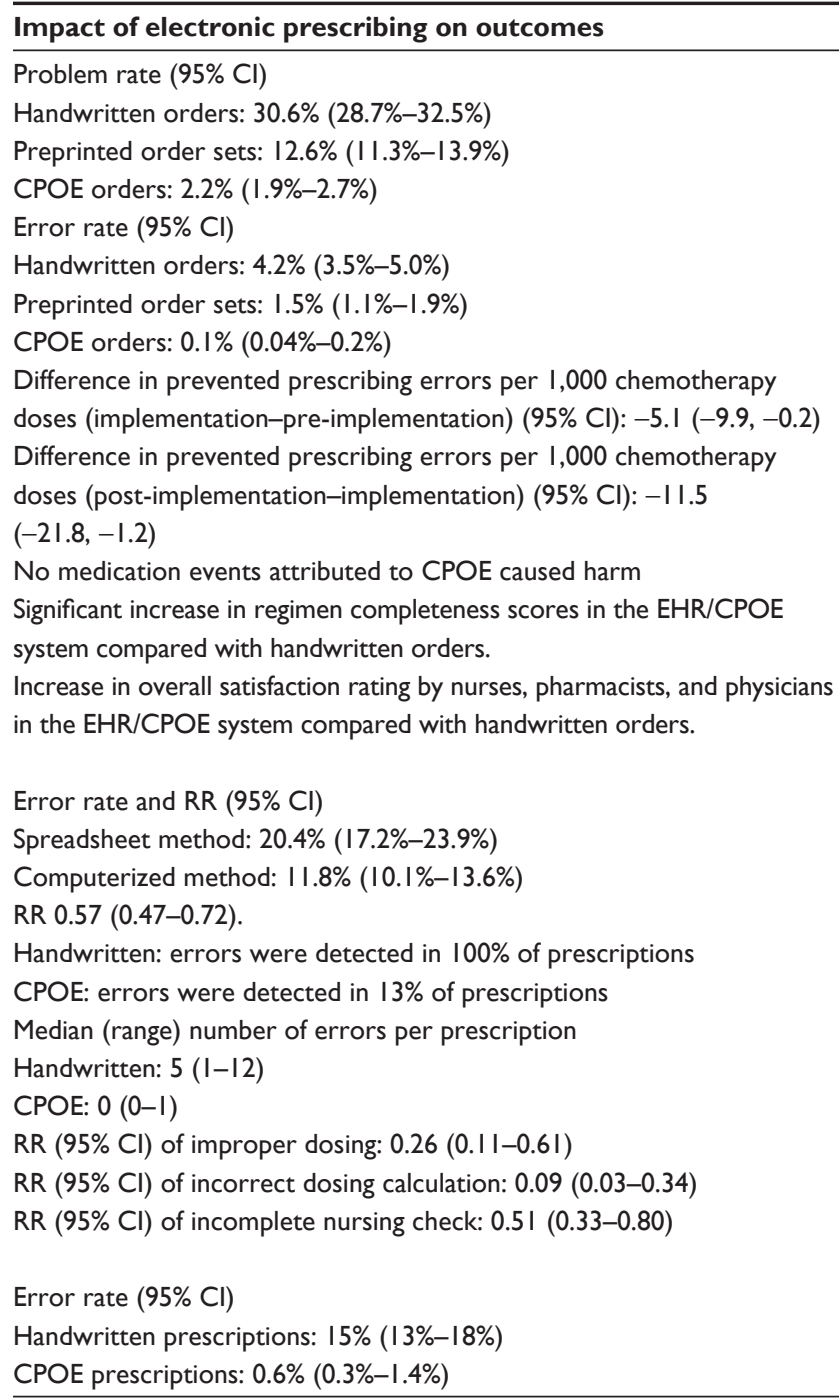

\section{Sample size and statistical analysis}

2,2। 6 handwritten; 2,480 preprinted order sets and 5, I42 CPOE order sets

Two-sample proportions test (chi-square test)

\author{
28,560 doses (pre-implementation) \\ 37,808 doses (implementation) \\ 43,206 doses (post-implementation) \\ Statistical comparisons were conducted using linear regression analysis
}

N/A

45 charts in the EHR/CPOE group and 45 charts in the handwritten group. Satisfaction survey was administered to 33 nurses, 8 pharmacists, and 5 physicians.

Statistical comparisons were conducted using two-sample proportions test (chi-square test)

314 spreadsheet prescriptions

I,339 computerized prescriptions

Chi-square test and RR for errors with computerized versus

spreadsheet prescribing estimated using Poisson regression

30 handwritten prescriptions

30 CPOE prescriptions

Two-sample proportions test (chi-square test)

I,250 handwritten prescriptions

I, II 6 CPOE prescriptions

940 handwritten prescriptions

527 CPOE prescriptions 
Table 3 Development of oral chemotherapy electronic prescribing interventions in hospital and ambulatory care settings

\begin{tabular}{|c|c|c|c|}
\hline Reference & Setting & $\begin{array}{l}\text { Form of chemotherapy } \\
\text { electronic prescribing }\end{array}$ & Safety and clinical decision support features \\
\hline Weingart et $\mathrm{al}^{|3|}$ & $\begin{array}{l}\text { Ambulatory } \\
\text { setting in } \\
\text { the USA }\end{array}$ & $\begin{array}{l}\text { Modification of an } \\
\text { existing CPOE system } \\
\text { by programming oral } \\
\text { chemotherapy agents }\end{array}$ & $\begin{array}{l}\text { CPOE is part of the EHR system. } \\
\text { Prescribing oral chemotherapy as a fixed dose, weight-based, or BSA-based dosing. } \\
\text { CDS alerts in the form of dose limit alerts. } \\
\text { Entry fields for diagnosis, intent of therapy, protocol, and cycle numbers. }\end{array}$ \\
\hline Collins and Elsaid 25 & $\begin{array}{l}\text { Inpatient } \\
\text { setting in } \\
\text { the USA }\end{array}$ & $\begin{array}{l}\text { Modification of an } \\
\text { existing CPOE system } \\
\text { by programming oral } \\
\text { chemotherapy agents }\end{array}$ & $\begin{array}{l}\text { CDS alerts as follows: } \\
\text { Alert is displayed that drug is a chemotherapy agent } \\
\text { Programmed drug-specific maximum dose alerts, and frequency options. } \\
\text { Default standard minimum dose, frequency, and duration. } \\
\text { Entry fields for cycle and protocol number. } \\
\text { Link to drug therapy guidelines and laboratory monitoring orders are suggested. }\end{array}$ \\
\hline
\end{tabular}

Abbreviations: CDS, clinical decision support; CPOE, computerized provider order entry; BSA, body surface area; EHR, electronic health records.

fields varied significantly, with the intent of therapy specified in approximately $13 \%$ of prescriptions and the patient's diagnosis listed in $46 \%$ of prescriptions. Prescribers used the free-text entry fields in $64 \%$ of all prescriptions to customize their prescriptions, clarify their dose strength selection, or their dosing modification rationale. The authors identified that doselimit safety alerts were triggered for $6 \%$ of all prescriptions entered following implementation of oral chemotherapy CPOE prescribing. Interestingly, in only $4 \%$ of prescriptions where a dose limit safety alert was triggered, physicians proceeded to abort the prescription, in contrast with $96 \%$ of alert-triggered prescriptions where physicians continued the prescription. Aborted prescriptions were most likely associated with a single oral chemotherapeutic agent, ie, temozolomide.

\section{Adoption of chemotherapy CPOE and usability challenges}

Adoption of chemotherapy CPOE is hampered by barriers and challenges that include financial cost, cultural challenges (eg, need for process change), technological challenges (eg, lack of system interoperability), and user-generated errors. $^{34,35}$ In a systematic analysis of published reports discussing barriers to CPOE implementation, the need for process changes was cited most frequently as a barrier to CPOE adoption. ${ }^{35}$ The second most cited reason for lack of CPOE adoption was the need for extensive training, which is required of all employees, followed by lack of high-quality studies documenting the efficacy of CPOE in reducing errors and improving quality of care. ${ }^{35}$ Combined, these three barriers represented $46 \%$ of cited CPOE adoption barriers. ${ }^{35}$ Current users of chemotherapy CPOE are faced with another set of challenges. In a recent study, a survey was administered to current chemotherapy CPOE users. ${ }^{36}$ The survey focused on understanding the challenges that institutions faced with implementation of CPOE and continue to face in using the CPOE system. ${ }^{36}$ Current $\mathrm{CPOE}$ users reported that they faced challenges related to standardization of workflow processes, CPOE system selection, and procurement. Current CPOE users also reported system usability issues following implementation. The most frequently cited usability issues included suitability of alerts and warnings, discrepancies in documentation and data entry, and lack of appropriate safeguards. These issues were reported by more than $50 \%$ of the CPOE users who responded to the survey. ${ }^{36}$

Table 4 Outcomes of oral chemotherapy electronic prescribing interventions in hospital and ambulatory care settings

\begin{tabular}{|c|c|c|c|c|}
\hline Reference & $\begin{array}{l}\text { Study } \\
\text { design }\end{array}$ & Outcomes of interest & $\begin{array}{l}\text { Impact of electronic } \\
\text { prescribing on outcomes }\end{array}$ & $\begin{array}{l}\text { Sample size and } \\
\text { statistical analysis }\end{array}$ \\
\hline Weingart et $\mathrm{al}^{31}$ & $\begin{array}{l}\text { Cohort } \\
\text { study }\end{array}$ & $\begin{array}{l}\text { Utilization of non-mandatory } \\
\text { entry fields, eg, diagnosis and } \\
\text { intent of therapy } \\
\text { Dose limit alerts } \\
\text { Prescriber actions following } \\
\text { alert generation }\end{array}$ & $\begin{array}{l}\text { Utilization of diagnosis field, } 46 \% \\
\text { Utilization of therapy intent field, I3\% } \\
\text { Dose limit alerts triggered } \\
\text { for } 6 \% \text { of prescriptions; } \\
4 \% \text { of prescriptions with dose } \\
\text { limit alerts were aborted }\end{array}$ & $\begin{array}{l}6,673 \text { prescriptions in the } \\
\text { post-implementation phase. } \\
\text { Statistical tests were } \\
\text { conducted using statistical } \\
\text { software but were not } \\
\text { specified }\end{array}$ \\
\hline Collins and Elsaid 25 & $\begin{array}{l}\text { Before } \\
\text { and after } \\
\text { design }\end{array}$ & $\begin{array}{l}\text { Prescribing error rate using } \\
\text { handwritten prescriptions } \\
\text { versus CPOE prescriptions }\end{array}$ & OR $0.31,95 \% \mathrm{Cl} 0.1 \mathrm{I}-0.89$ & $\begin{array}{l}412 \text { handwritten prescriptions } \\
\text { I } 26 \text { CPOE prescriptions } \\
\text { Fisher's exact test }\end{array}$ \\
\hline
\end{tabular}

Abbreviations: $\mathrm{Cl}$, confidence interval; $\mathrm{CPOE}$, computerized provider order entry; $\mathrm{OR}$, odds ratio. 


\section{Chemotherapy CPOE system design and implementation}

Organizations considering implementation of electronic prescribing for chemotherapy agents should critically evaluate their current chemotherapy medication use processes and identify areas where safety and quality can be improved. Conducting a failure modes and effects analysis (FMEA) can proactively uncover steps at which the chemotherapy medication use process may fail and the probability of that occurring. ${ }^{25,37,38}$ In addition to FMEA, a formal redesign of the process and system analysis should take place. It is essential that process redesign and FMEA analyses involves a multidisciplinary team directly involved in the prescribing, dispensing, preparation, administration, and monitoring of chemotherapy agents. Results of these analyses should guide the design approach for CPOE and CDS. ${ }^{10,25,31}$

Given that chemotherapy regimens are complex, with multiple drugs with unique dosing and sequencing requirements, electronic chemotherapy order set templates should be carefully designed and tested repeatedly for accuracy prior to implementation. Additionally, these order sets should have enough flexibility to allow prescribers to modify them based on patient status. CDS is an important consideration of CPOE design. The CDS features of chemotherapy CPOE should include alerts for drug-drug interactions, duplicate therapy alerts, dose limit alerts, and the ability to direct prescribers to order appropriate laboratory tests, supportive care, and hydration requirements. ${ }^{10,39}$ The choice and programming of CDS features should have the goal of minimizing "alert fatigue", and it is recommended that CDS alerts be reviewed on a regular basis with the goal of removing those alerts with little clinical value and introducing new alerts that are deemed important. ${ }^{10,39}$ Implementation of the CPOE system should occur in a way and at a pace that does not compromise the safety of the medication use process. A phased implementation approach has been reported in a number of studies. ${ }^{10,15,16,21}$ Training must be provided to all employees. The organization should have a clear plan for the method of training that it will provide prior to implementation of CPOE. In one study, the organization used a combination of online training modules and instructor-led training sessions. ${ }^{10}$ Following implementation, institutions should develop and use a risk assessment process to identify and evaluate unanticipated consequences and new CPOE-generated errors. ${ }^{40}$ The knowledge generated from these assessments should be used to further modify and refine the CPOE system as part of an ongoing continuous quality improvement process.

\section{Conclusion}

Adoption of chemotherapy CPOE remains suboptimal. There is a limited number of published studies that report on the design, implementation, and outcomes of infusional chemotherapy in the ambulatory and inpatient setting. There are even fewer studies that discuss enhancing the safety of oral chemotherapy via electronic prescribing. Most published studies report a reduction in prescribing errors; most significantly those involving dose calculation or adjustment. Some studies have focused on delineating the impact of computerized chemotherapy prescribing on the chemotherapy process itself. In this regard, electronic prescribing was associated with more complete documentation. The conclusion that electronic chemotherapy prescribing reduces prescribing errors is limited by the methodological limitations of the designs of most studies and the short follow-up period following CPOE implementation. There is a heightened need to develop guidelines and disseminate examples of best practice to guide interested organizations through the process of selection, procurement, and implementation of an electronic prescribing system. There is also a significant need to conduct well designed studies to investigate the effects of chemotherapy electronic prescribing systems on the overall safety and quality of cancer care.

\section{Disclosure}

The authors report no conflicts of interest in this work.

\section{References}

1. Walsh KE, Dodd KS, Seetharaman K, et al. Medication errors among adults and children with cancer in the outpatient setting. J Clin Oncol. 2009;27:891-896.

2. Gandhi TK, Bartel SB, Shulman LN, et al. Medication safety in the ambulatory chemotherapy setting. Cancer. 2005;104:2477-2483.

3. Bates DW. Preventing medication errors: a summary. Am J Health Syst Pharm. 2007;64(14 Suppl 9):S3-S9.

4. Keers RN, Williams SD, Cooke J, Ashcroft DM. Causes of medication administration errors in hospitals: a systematic review of quantitative and qualitative evidence. Drug Saf. 2013;36:1045-1067.

5. Pedersen CA, Schneider PJ, Scheckelhoff DJ. ASHP national survey of pharmacy practice in hospital settings: dispensing and administration 2011. Am J Health Syst Pharm. 2012;69:768-785.

6. HealthIT.gov. Update on the adoption of health information technology and related efforts to facilitate the electronic use and exchange of health information. Available from: http:/www.healthit.gov/policyresearchers-implementers/health-it-adoption-rates. Accessed March 7, 2015.

7. Wright A, Henkin S, Feblowitz J, McCoy A, Bates DW, Sittig D. Early results of the meaningful use program for electronic health records. N Engl J Med. 2013;368:779-780.

8. Nuckols TK, Smith-Spangler C, Morton SC, et al. The effectiveness of computerized order entry at reducing preventable adverse drug events and medication errors in hospital settings: a systematic review and metaanalysis. Syst Rev. 2014;3:56. 
9. Devine EB, Hansen RN, Wilson-Norton JL, et al. The impact of computerized provider order entry on medication errors in a multispecialty group practice. J Am Med Inform Assoc. 2010;17:78-84.

10. Hoffman JM, Baker DK, Howard SC, Laver J, Shenep J. Safe and successful implementation of CPOE for chemotherapy at a children's cancer center. J Natl Compr Canc Netw. 2011;9:S36-S50.

11. Ash JS, Sittig DF, Poon EG, Guappone K, Campbell E, Dykstra RH. The extent and importance of unintended consequences related to computerized provider order entry. J Am Med Inform Assoc. 2007;14: 415-423.

12. Koppel R, Metlay JP, Cohen A, et al. Role of computerized physician order entry systems in facilitating medication errors. JAMA. 2005;293: 1197-1203.

13. Han YY, Carcillo JA, Venkataraman ST, et al. Unexpected increased mortality after implementation of a commercially sold computerized physician order entry system. Pediatrics. 2005;116:1506-1512.

14. Meisenberg BR, Wright R, Brady-Copertino CJ. Reduction in chemotherapy order errors with computerized physician order entry. J Oncol Pract. 2014;10:e5-e9.

15. Harshberger C, Harper A, Carro GW, et al. Outcomes of computerized physician order entry in an electronic health record after implementation in an outpatient oncology setting. J Oncol Pract. 2011;7: 233-237.

16. Brockstein B, Hensing T, Carro GW, et al. Effect of an electronic health record on the culture of an outpatient medical oncology practice in a four-hospital integrated health care system: 5-year experience. J Oncol Pract. 2011;7:e20-e24.

17. Kim G, Chen A, Arceci R, Mitchell S, Kokoszka M, Lehmann C. Error reduction in pediatric chemotherapy. Arch Pediatr Adolesc Med. 2006;160:495-498.

18. Small M, Barrett A, Price GM. The impact of computerized prescribing on error rate in a department of oncology/hematology. J Oncol Pharm Pract. 2008;14:181-187.

19. Huertas Fernández M, Baena-Canada JM, Martinez Bautista M, Arriola Arellano E, Garcia Palaois M. Impact of computerised chemotherapy prescriptions on the prevention of medication errors. Clin Transl Oncol. 2006;8:821-825.

20. Voeffray M, Pannatier A, Stupp R, Fucino N, Leyvraz S, Wasserfallen JB. Effect of computerization on the quality and safety of chemotherapy prescription. Qual Saf Health Care. 2006;160:495-498.

21. Elsaid KA, Truong T, Monckeberg M, McCarthy H, Butera J, Collins C. Impact of electronic chemotherapy order forms on prescribing errors at an urban medical centers: results from an interrupted time-series analysis. Int J Qual Health Care. 2013;25:656-663.

22. Scholler R, Leveque D. Molecular pharmacokinetic determinants of anticancer kinase inhibitors in humans. Oncol Rev. 2011;5:77-92.

23. Rittenberg CN. Meeting educational needs and enhancing adherence of patients receiving oral cancer agents through use of the MASCC oral agent teaching tool. Eur Oncol Haematol. 2012;8:97-100.
24. Weingart SN, Spencer J, Buia S, et al. Medication safety of five oral chemotherapies: a proactive risk assessment. J Oncol Pract. 2011;7: 2-6.

25. Collins CM, Elsaid KA. Using an enhanced oral chemotherapy computerized provider order entry system to reduce prescribing errors and improve safety. Int J Qual Health Care. 2011;23:36-43.

26. Bartel SB. Safe practices and financial considerations in using oral chemotherapeutic agents. Am J Health Syst Pharm. 2007;64(9 Suppl 5): S8-S14.

27. Weingart SN, Flug J, Brouillard D, et al. Oral chemotherapy safety practices at US cancer centers: questionnaire survey. BMJ. 2007;334:407.

28. Ahmed N, Simanovski V, Hertz S, Klaric G, Kaizer L, Krzyzanowska MK. Oral chemotherapy practices at Ontario cancer centres. J Oncol Pharm Pract. April 8, 2014. [Epub ahead of print].

29. Weingart SN, Toro J, Spencer J, et al. Medication errors involving oral chemotherapy. Cancer. 2010;116:2455-2464.

30. Moore TJ, Walsh CS, Cohen MR. Reported medication errors associated with methotrexate. Am J Health Syst Pharm. 2004;61:1380-1384.

31. Weingart SN, Mattsson T, Zhu J, Shulman LN, Hassett M. Improving electronic oral chemotherapy prescription; can we build a safer system? J Oncol Pract. 2012;8:e168-e173.

32. Dean B, Barber N, Schachter M. What is a prescribing error? Qual Health Care. 2000;9:232-237.

33. Ghaleb M, Barber N, Dean F, et al. What constitutes a prescribing error in pediatrics? Qual Saf Health Care. 2005;14:352-357.

34. Charles K, Cannon M, Hall R, Coustasse A. Can utilizing a computerized provider order entry (CPOE) system prevent hospital medical errors and adverse drug effects. Perspect Health Inf Manag. 2014;11:1b.

35. Kruse CS, Goetz K. Summary and frequency of barriers to adoption of CPOE in the US. J Med Syst. 2015;39:15.

36. Jeon J, Taneva S, Kukreti V, et al. Toward successful migration to computerized physician order entry for chemotherapy. Curr Oncol. 2014;21:e221-e228.

37. [No authors listed]. An introduction to FMEA. Using failure modes and effects analysis. Health Devices. 2002;31:223-226.

38. Weingart SN, Spencer J, Buia S, et al Medication safety of five oral chemotherapies: a proactive risk assessment. J Oncol Pract. 2011;7: 2-6.

39. ASHP Section of Pharmacy Informatics and Technology. ASHP guidelines on pharmacy planning for implementation of computerized provider order entry systems in hospitals and health systems. Am J Health Syst Pharm. 2011;68:e9-e31.

40. Kukreti V, Cosby R, Cheung A, Lankshear S. Computerized prescriber order entry in the outpatient oncology setting: from evidence to meaningful use. Curr Oncol. 2014;21:e604-e612.
Integrated Pharmacy Research and Practice

\section{Publish your work in this journal}

Integrated Pharmacy Research and Practice is an international, peer-reviewed, open access, online journal, publishing original research, reports, reviews and commentaries on all areas of academic and professional pharmacy practice. This journal aims to represent the academic output of pharmacists and pharmacy practice with particular focus on integrated care. All papers are carefully peer reviewed

\section{Dovepress}

to ensure the highest standards as well as ensuring that we are informing and stimulating pharmaceutical professionals. The manuscript management system is completely online and includes a very quick and fair peer-review system, which is all easy to use. Visit http://www.dovepress.com/testimonials.php to read real quotes from published authors. 